\title{
Empirical Noise Modelling in Power Line Communication Systems
}

\author{
Guoping Chen, Gonghai Yang* \\ School of Optoelectronic Engineering, Chongqing University of Posts and Telecommunications, Chongqing \\ 400065, China.
}

* Corresponding author. Tel.: (+86)18883283650; email: yangghstu@foxmail.com

Manuscript submitted June 22, 2018; accepted September 18, 2018.

doi: 10.17706/ijcce.2018.7.4.119-127

\begin{abstract}
Power line communication (PLC) systems are contaminated with complicated noise. Five types of noise in power line communication systems are introduced in this paper. According to their properties, these noises are mainly divided into two types that are background noise and impulsive noise. Noise modelling approaches for background noise and impulsive noise are reviewed in this paper. A frequency-domain based model is applied to describe the properties of background noise and a frequency decreasing power spectral density (PSD) is revealed based on this model. The values of PSD are between $-140 \mathrm{dBm} / \mathrm{Hz}$ and $-80 \mathrm{dBm} / \mathrm{Hz}$. A time-domain approach which is based on partitioned Markov chain is applied to describe the time behaviour of impulsive noise. Simulation results showed this model is suitable for the description and modelling of impulsive noise. This model revealed that impulsive noise rarely exceeds a few milliseconds.
\end{abstract}

Key words: Power line communication, noise modelling, power spectral density, Markov chain.

\section{Introduction}

Power line communication reuses the existing power line network whose primary purpose is to deliver electric power, for data communications [1]. Power lines are ubiquitous in cities and houses, which makes PLC a promising technology for data transmission in smart city and smart home.

PLC can be categorised into narrowband PLC and broadband PLC over frequency band. The narrowband PLC operates between $3 \mathrm{kHz}$ to $500 \mathrm{kHz}$ while the broadband PLC operates between $1.8 \mathrm{MHz}$ to $250 \mathrm{MHz}$ [1], [2]. In all communication systems, noise characteristics are important parameters to describe the nature of channel interference. When to design communication systems, eliminating noise is an efficient method to improve the performance of communication. Mathematical models are fundamental tools to reveal the characteristics of noise in communication systems. In the design and analysis of conventional communication systems, stationary additive white Gaussian noise (AWGN) is often used as a model of noise. In PLC systems, however, noise is quite different from the stationary AWGN. Noise in PLC systems results from various devices connected to the grid and often are non-Gaussian noise.

Power line network is complex and usually in a harsh environment, which makes it a great challenge to design high performance PLC systems. In order to understand the effect of noise on the performance of PLC systems, it is important to analyse its structure both in time-domain and frequency-domain. Many researchers have contributed a lot on the PLC noise research and obtained a lot of remarkable achievements. In [3], a mathematically tractable and accurate model narrowband power line noise based on 
experiment measurement was introduced and the noise was expressed as a Gaussian process whose instantaneous variance was a periodic time function. In [4], the characteristic and mathematical model of colored background noise was studied based on neural network and it showed that artificial neural network modelling method was efficient for modelling the colored background noise in PLC systems. In [5], some circuits were set up to measure the noise on the power line and then the power line colored background noise model, narrowband noise model and burst noise model were set up respectively based on the measured noise data.

The rest of this paper is organised as follows. In Section 2, classification of noise in PLC systems will be discussed. In Section 3, approaches of noise modelling and simulation results will be discussed. In Section 4, a conclusion will be made to conclude this paper.

\section{Noise Classification}

Noise in PLC systems consists of background noise and impulsive noise. In general, the former is stationary whereas the latter is nonstationary. From many researchers' experimental measurements and studies, for example, in [1], [2], [4], [6], [7], noise in PLC can be categorised into five types that are colored background noise, narrowband noise, periodic impulsive noise asynchronous to the main frequency, periodic impulsive noise synchronous to the main frequency, asynchronous impulsive noise. All these noise are shown in Fig. 1 and their sources properties and effects are summarised in Table 1.

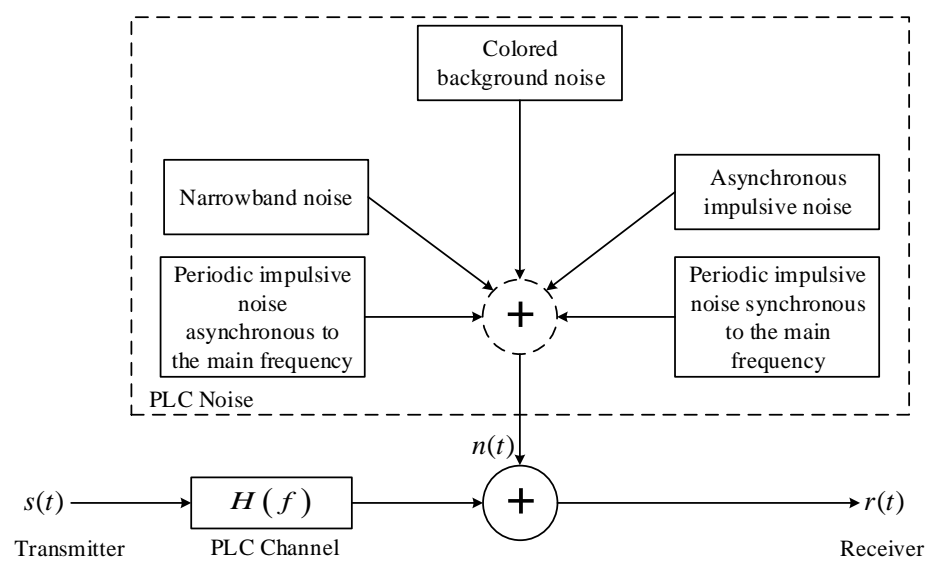

Fig. 1. Noise components in PLC systems.

Table 1. Noise Types, Sources, Properties and Effects

\begin{tabular}{|c|c|c|}
\hline Noise & Noise sources & Properties and effects \\
\hline Colored background noise & $\begin{array}{l}\text { summation of numerous noise with } \\
\text { low power }\end{array}$ & $\begin{array}{l}\text { a frequency decreasing PSD, } \\
\text { slowly varying parameters }\end{array}$ \\
\hline Narrowband noise & medium and short wave broadcast & $\begin{array}{l}\text { large PSD, narrow bandwidth, } \\
\text { varying slowly }\end{array}$ \\
\hline $\begin{array}{l}\text { Periodic impulsive noise } \\
\text { asynchronous to the main } \\
\text { frequency }\end{array}$ & switched power supplies & $\begin{array}{l}\text { a repetition rate between } 50 \mathrm{~Hz} \\
\text { and } 200 \mathrm{kHz} \text {, a discrete line } \\
\text { spectrum }\end{array}$ \\
\hline $\begin{array}{l}\text { Periodic impulsive noise } \\
\text { synchronous to the main } \\
\text { frequency }\end{array}$ & $\begin{array}{l}\text { power supplies, switch of rectifier } \\
\text { diodes }\end{array}$ & $\begin{array}{l}\text { a repetition of } 50 \text { or } 100 \mathrm{~Hz} \text {, a PSD } \\
\text { decreasing with frequency }\end{array}$ \\
\hline $\begin{array}{l}\text { Asynchronous impulsive } \\
\text { noise }\end{array}$ & switching transients & $\begin{array}{l}\text { large PSD, random occurrence, a } \\
\text { duration of some microseconds } \\
\text { up to a few milliseconds }\end{array}$ \\
\hline
\end{tabular}


From Table 1, the former two types of noise usually vary slowly over time and remain stationary, so they can be recognized as background noise. However, the latter three types of noise change rapidly and are cyclostationary or bursty or aperiodic, so they can be recognized as impulsive noise. In contrast to conventional AWGN, these noises have different models and properties, therefore, Gaussian process is no longer an accurate model to analyse these noises. Among impulsive noise, the aperiodic component is the most unpredictable component and it is due to the connection and disconnection of appliances from the power delivery network. The amplitude of the aperiodic can be significantly larger than that of other impulsive noise components [6]. Besides the amplitude, the aperiodic noise is typically described by the duration and the inter-arrival time [8].

\section{Noise Models and Simulations}

\subsection{Background Noise Modelling}

The PLC colored background noise is usually modelled with the stationary Gaussian colored process having a frequency decreasing PSD profile, according to a polynomial or exponential function of frequency. It is common to use autoregression (AR) model to model PLC colored background noise. In this process, AR model can be regarded as a finite impulse response (FIR) filter to modify the input sequences to get the output sequences that is wanted. Giving a Gaussian white noise sequence as the input of the AR model then its output can be regarded as the colored background noise. This can be shown in Fig. 2.

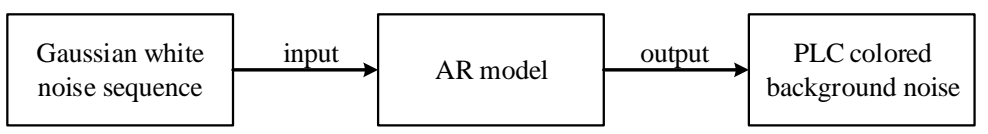

Fig. 2. Schematic diagram of colored background noise modelling.

The colored background noise has a frequency decreasing PSD profile. In general, the values of PSD are between $-140 \mathrm{dBm} / \mathrm{Hz}$ and $-80 \mathrm{dBm} / \mathrm{Hz}$. Its PSD can be expressed as (1) that is an exponential function of frequency.

$$
S_{C B N}(f)=A_{0}+A_{1} \cdot e^{-\frac{f}{f_{0}}}
$$

From (1), the model of colored background noise is described by three parameters $A_{0}, A_{1}, f_{0}$. Among them, $A_{0}$ is the constant noise power density, $A_{1}$ is the value of PSD when the frequency is 0 and $f_{0}$ is the fading rate. In [9], the values of parameters $A_{0}, A_{1}, f_{0}$ have been given. According to the model expressed by (1), the PSD of colored background noise is shown in Fig. 3.

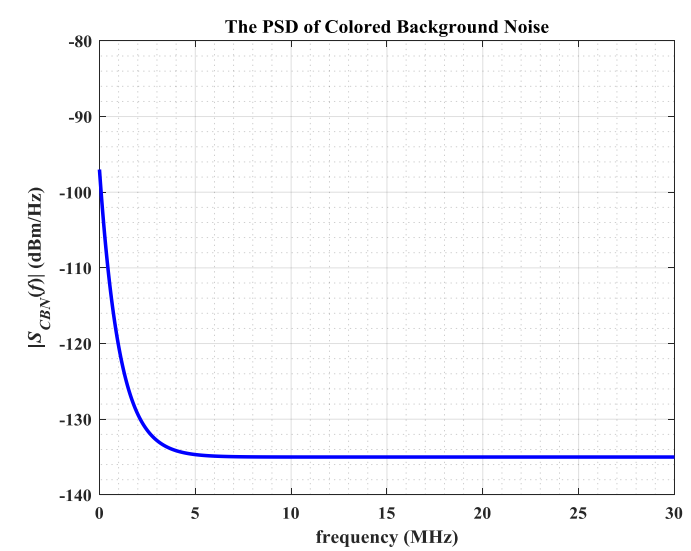

Fig. 3. The PSD of colored background noise. 
Fig. 3 shows that the PSD of colored background noise decrease exponentially up to $5 \mathrm{MHz}$. Above $5 \mathrm{MHz}$ the noise floor is approximately flat that is $-135 \mathrm{dBm} / \mathrm{Hz}$. This reveals the colored background noise has greater effects on PLC systems at low frequency.

Another component of background noise is narrowband noise which results from radio signals. It has large PSD and narrow bandwidths. Generally, a parametric Gaussian function expressed by (2) has been used for the approximation of the narrowband noise [9]. The model consists of three parameters $A_{\mathrm{k},}, f_{0, \mathrm{k}}$ and $B_{\mathrm{k}}$. They represent the amplitude, the center frequency and the bandwidth of the Gaussian function respectively.

$$
S_{N N}^{(s, k)}(f)=A_{k} \cdot e^{-\frac{\left(f-f_{0, k}\right)^{2}}{2 \cdot B_{k}^{2}}}
$$

The PSD of the narrowband noise based on the model described by (2) is shown in Fig. 4. At some deterministic frequencies, the noise floor level is very large.

Combining (1) with (2) then the model of PLC background noise expressed by (3) is obtained. This model is controlled by seven parameters $\left(A_{0}, A_{1}, f_{0}, A_{\mathrm{k}}, f_{0, \mathrm{k}}, B_{\mathrm{k}}, N\right)$. Every one of these parameters should be described by probability density function (PDF). This function reflects the statistical characteristic of the model.

$$
S_{B N}(f)=A_{0}+A_{1} \cdot e^{-\frac{f}{f_{0}}}+\sum_{k=1}^{N} A_{k} \cdot e^{-\frac{\left(f-f_{0, k}\right)^{2}}{2 \cdot B_{k}^{2}}}
$$

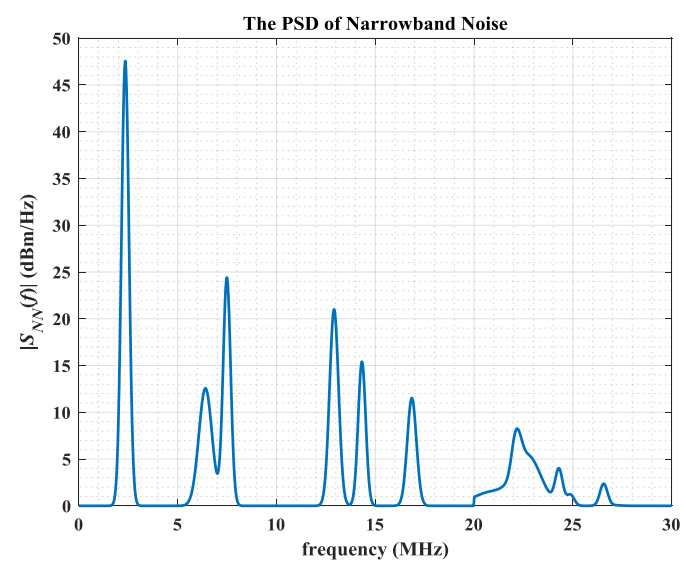

Fig. 4. The PSD of narrowband noise.

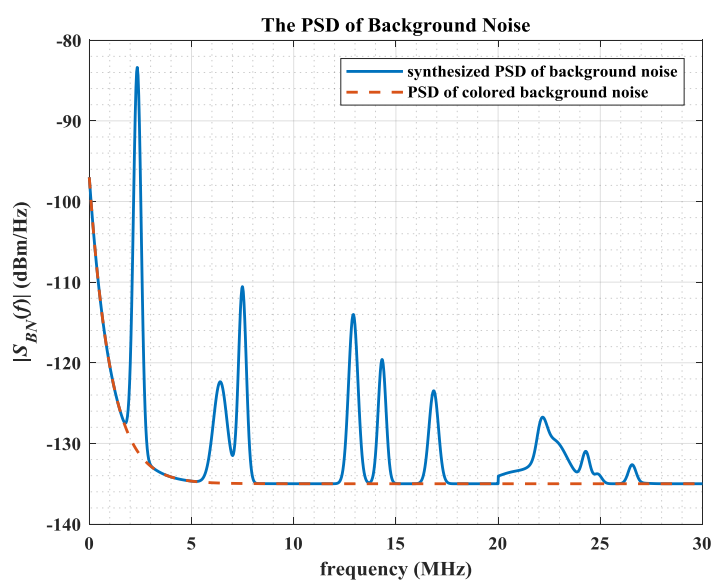

Fig. 5. The PSD of synthesized background noise. 
The PSD of synthesized background noise is shown in Fig. 5. This figure reveals that the noise floor level can be approximately regarded as a constant at upper frequency band. In general, the narrowband noise has stronger disturbance than the colored background noise.

\subsection{Impulsive Noise Modelling}

Impulsive noise is another type of noise existing in PLC systems. Generally, it is unpredictable and its amplitude is much higher than the background noise. Three important characteristic parameters of impulsive noise are: amplitude, width and inter-arrival time (IAT) [8]. Comparing with frequency-domain background noise modelling method, the impulsive noise is typically in time-domain. In [10], Middleton's class A distribution is suggested to fit the amplitude of the impulsive noise. In [11], Nakagami-m distribution is proposed to fit the amplitude of the impulsive noise. Another model which is based on Markov chain is also proposed to model the impulsive noise.

The shape of impulsive noise is often similar to damped sinusoid signals or superimposed sinusoid signals. Fig. 6 shows two types of impulsive noise in time-domain.
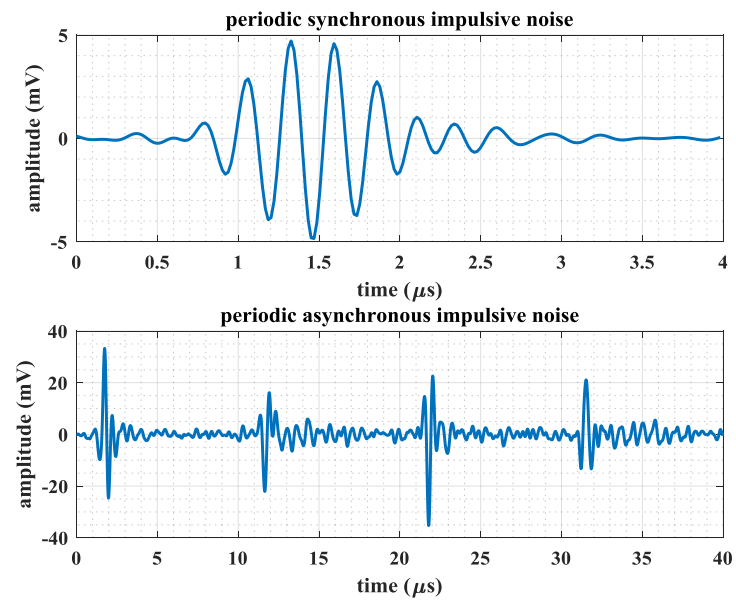

Fig. 6. Time-domain waveform of impulsive noise.

In order to study the time behaviour of impulsive noise, the assumption of rectangular envelopes has been proposed in [7]. This rectangular envelopes can be described by three characteristic parameters, for example, impulse width $t_{w}$, arrival time $t_{\text {arr, }}$ and inter-arrival time $t_{\text {IAT. }}$ The time-domain impulse representation and rectangular envelope with characteristic parameters is shown in Fig. 7.

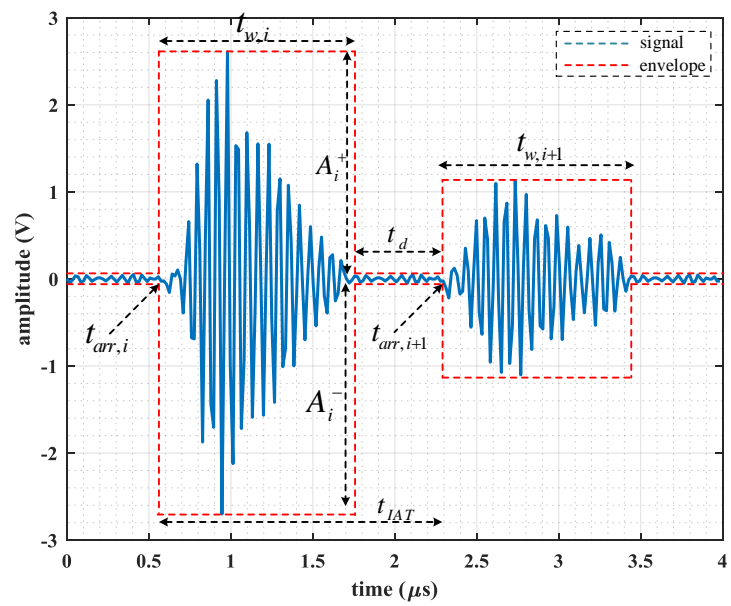

Fig. 7. Time-domain impulse representation and envelope with characteristic parameters. 
From Fig. 7 the distance between two impulse events is described by the inter-arrival time $t_{\mathrm{IAT}}$ or impulse distance $t_{\mathrm{d}}$ and their relation can be described by (4).

$$
t_{\mathrm{IAT}}=t_{w}+t_{d}=t_{\mathrm{arr}, i+1}-t_{\mathrm{arr}, i}
$$

A generalized impulse function $\operatorname{imp}(t)$ with unit amplitude and unit width can be used to describe the train of impulses $n_{\text {imptrain }}(t)$ described by (5).

$$
n_{\text {imprain }}(t)=\sum_{i=1}^{N} A_{i} \cdot \operatorname{imp}\left(\frac{t-t_{\text {arr }, \mathrm{i}}}{t_{w, i}}\right)
$$

The parameters $A, t_{\mathrm{w}}$ and $t_{\mathrm{arr}}$ are random variables, whose statistical properties can be investigated by measurements. In [7], two useful parameters called impulse rate and disturbance ratio were introduced to characterize the impulse noise scenario and its behaviour over time, and they are described by (6) and (7) respectively.

$$
r_{\text {imp }}=\frac{N_{\text {imp }}}{T_{\text {win }}}
$$

As described by (6), $N_{\text {imp }}$ denotes the number of impulses, occurring within an observation window $T_{\text {win. }}$.

$$
\text { disturbance_ratio }=\frac{\sum_{i=1}^{N_{\text {imp }}} t_{w, i}}{T_{\text {win }}}
$$

Equation (7) is defined by the ratio of the sum of the widths of all the impulse events occurring within an observation window $T_{\text {win }}$. According to this equation, the percentage of impulse events can be approximately evaluated within an observation window. In [7], a partitioned Markov chain was proposed to discuss the impulse widths and inter-arrival time which characterize the impulse events over time. Fig. 8 shows the diagram of partitioned Markov chain.

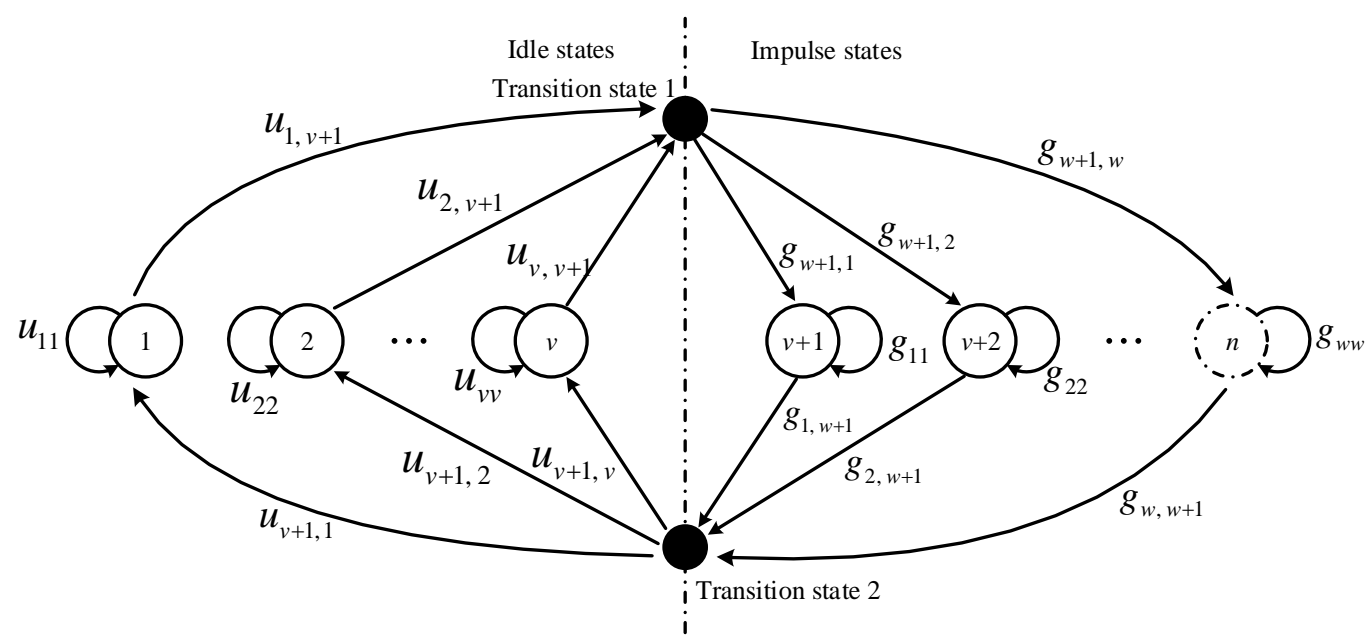

Fig. 8. Partitioned Markov chain for the representation of asynchronous impulse events.

In Fig. 8, there are two main states that are idle states and impulse states. While $n$ sub-states are included in these two main states, among them $v$ states represent the idle states where no impulse events occur, and the other $w(w=n-v)$ states represent the impulse states where an impulse event occurs. Each sub-state in idle states may stay the current state or transferring to impulse states, this mechanism still keeps the same for each sub-state in impulse states. In order to describe the statistical properties of Markov chain, a 
transition probability matrix $P$ defined by (8) is proposed [7].

$$
P=\left[\begin{array}{cccc}
p_{1,1} & p_{1,2} & \cdots & p_{1, n} \\
p_{2,1} & p_{2,2} & \ddots & \vdots \\
\vdots & \ddots & \ddots & p_{n-1, n} \\
p_{n, 1} & \cdots & p_{n, n-1} & p_{n, n}
\end{array}\right]
$$

The element $p_{\mathrm{i}, \mathrm{j}}$ in $P$ denotes the transition probability from the $i$ th state to the $j$ th state. For partitioned Markov chain, the idle states can be described by transition probability matrix $U$ defined by (9), and the impulse states can be described by transition probability matrix $G$ defined by (10) [7].

$$
U=\left[\begin{array}{ccccc}
u_{1,1} & 0 & \cdots & 0 & u_{1, v+1} \\
0 & u_{2,2} & \ddots & \vdots & u_{2, v+1} \\
\vdots & \ddots & \ddots & 0 & \vdots \\
0 & \cdots & 0 & u_{v, v} & u_{v, v+1} \\
u_{v+1,1} & u_{v+1,2} & \cdots & u_{v+1, v} & 0
\end{array}\right]
$$

and

$$
G=\left[\begin{array}{ccccc}
g_{1,1} & 0 & \cdots & 0 & g_{1, w+1} \\
0 & g_{2,2} & \ddots & \vdots & g_{2, w+1} \\
\vdots & \ddots & \ddots & 0 & \vdots \\
0 & \cdots & 0 & g_{w, w} & g_{w, w+1} \\
g_{w+1,1} & g_{w+2,2} & \cdots & g_{w+1, w} & 0
\end{array}\right]
$$

In matrix $U$, the diagonal elements denote the probabilities of staying the idle states and the elements in the last column denote the probabilities of transferring from the idle states to the impulse states. The elements in the last row denote which sub-state will be in the idle state when there a state jumps from the impulse state. The meaning of elements in $G$ can be explained by the same way.

As discussed above, the IAT and impulse width can be applied to evaluate the properties of impulse events over time. According to [7], the complimentary probability density functions (CPDF) of IAT and impulse width were introduced. The CPDF denotes the probability $P$ of a random variable $X$ exceeding a given value $x$ : $\operatorname{cpf}(x)=P(X>x)$. The CPFD of IAT and impulse width are defined by (11) and (12) respectively.

$$
\begin{aligned}
& c p f_{\mathrm{IAT}}=1, \quad k=0 \\
& c p f_{\mathrm{IAT}}=\sum_{j=1}^{v} u_{v+1, j} \cdot u_{j, j}^{k}, \quad k=1,2,3, \ldots
\end{aligned}
$$

and

$$
\begin{aligned}
c p f_{w} & =1, \quad k=0 \\
c p f_{w} & =\sum_{j=1}^{w} g_{w+1, j} \cdot g_{j, j}^{k}, \quad k=1,2,3, \ldots
\end{aligned}
$$

where $k$ denotes the time instants.

By applying a seven states partitioned Markov chain model where $v=5$ and $w=2$, the ability of this model to describe the statistical properties of impulse events is presented and discussed. The result of $c p f_{\mathrm{d}}$ according to (11) is plotted into Fig. (9) together with a simulation result of model parameters using Metropolis-Hasting (MH) algorithm where the sampling time is $50 \mu s$. 

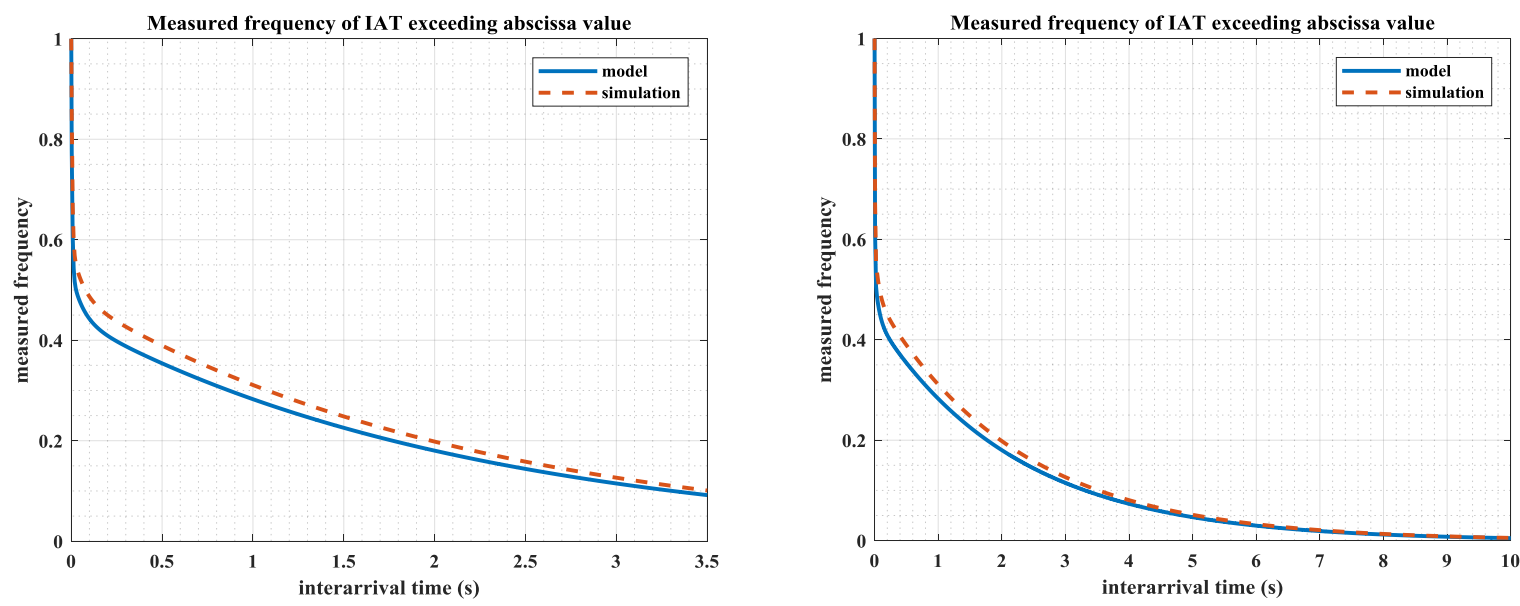

Fig. 9. The cpfd of IAT: Model and simulation.

The result of $c p f_{w}$ according to (12) is plotted into Fig. (10) together with a simulation result of model parameters using Metropolis-Hasting ( $\mathrm{MH})$ algorithm where the sampling time is $50 \mu s$.
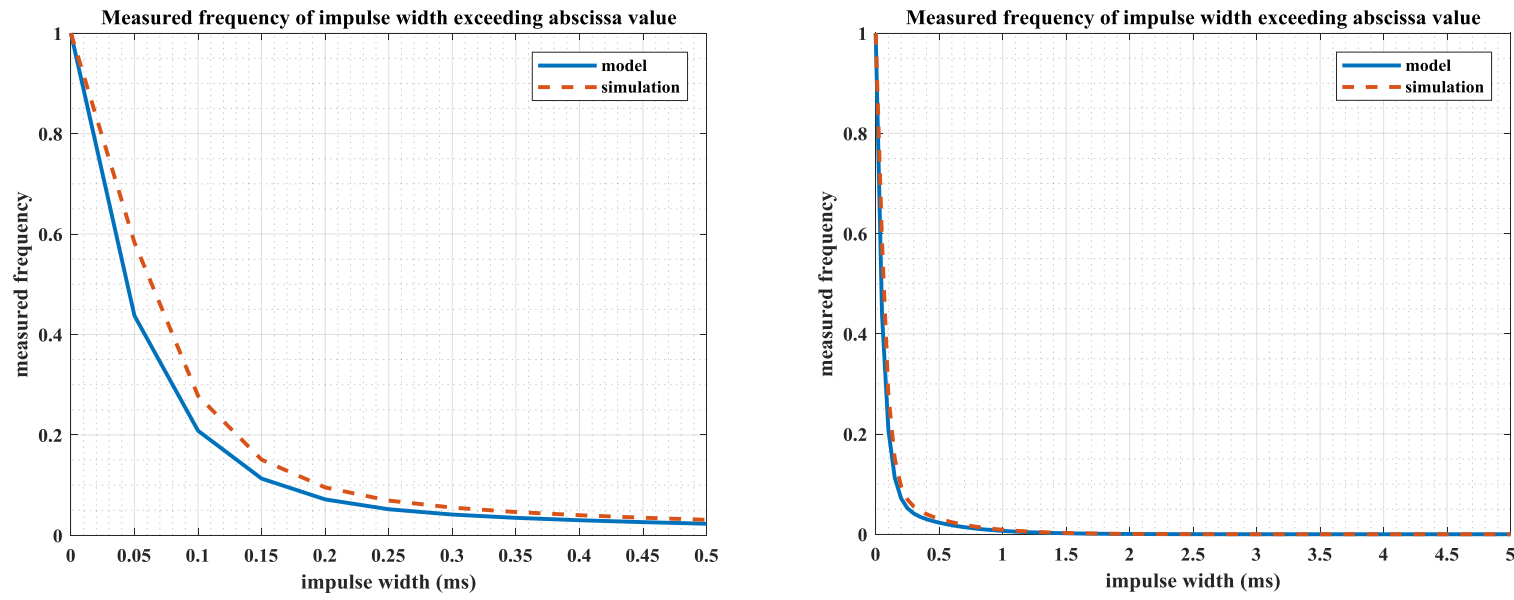

Fig. 10. The $c p f_{w}$ of impulse width: model and simulation.

From the simulations presented above, it is obvious that the simulation fits well with the theoretical model. This case illustrated that the presented model based on partitioned Markov chain is suitable for the description and modelling of the time behaviour of asynchronous impulsive noise in power line communication systems. Based on the analysis of the time behaviour of impulse width, it can be concluded that typical impulse events rarely exceeds a few milliseconds.

\section{Conclusion}

Noise is one of the most crucial factor degrading high-speed and robust data transmission systems. Noise in PLC systems cannot be regarded as AWGN that is widely used in many other communication systems. Power line network is contaminated with complicated noise. It is significant to analyse and model noise in PLC systems. Some efficient noise modelling approaches are reviewed in this paper. Background noise is modelled in frequency-domain and the result shows it has a frequency decreasing PSD which revealed the stronger interference in lower frequency band. Whereas the time behaviour of impulsive noise is analysed based on a partitioned Markov chain. The result shows a partitioned Markov chain is an efficient approach to model asynchronous impulse events in power line channels. The analysis shows that typical impulse events rarely exceeds a few milliseconds. 


\section{References}

[1] Lampe, L., Tonello, A. M., \& Swart, T. G. (2016). Power Line Communications: Principles, Standards and Applications from Multimedia to Smart Grid (2nd ed.). West Sussex: John Wiley \& Sons Ltd.

[2] Tan, B. (2013). Channel Modelling and Relay for Powerline Communications. PhD doctor of philosophy dissertation, University of Edinburgh, Edinburgh.

[3] Katayama, M., Yamazato, T., \& Okada, H. (2006). A mathematical model of noise in narrowband power line communication systems. IEEE Journal on Selected Areas in Communications, 24(7), 1267-1276.

[4] Ma, Y., Liu, K., Zhang, Z., et al. (2010). Modeling the colored background noise of power line communication channel based on artificial neural network. Proceedings of the 19th Annual Wireless and Optical Communications Conference (pp. 1-4).

[5] Zhang, Z., Sun, Y., Wang, D., et al. (2017). Modeling and research of noise characteristics for low voltage power line channel in OFDM communication system. Proceedings of the 2017 9th International Conference on Intelligent Human-Machine Systems and Cybernetics (pp.116-120).

[6] Cano, C., Pittolo, A., Malone, D., et al. (2016). State of the art in power line communications: From the applications to the medium. IEEE Journal on Selected Areas in Communications, 34(7), 1935-1952.

[7] Zimmermann, M., \& Dostert, K. (2002). Analysis and modeling of impulsive noise in broad-band powerline communications. IEEE Transactions on Electromagnetic Compatibility, 44(1), 249-258.

[8] Chan, M. H. L., \& Donaldson, R. W. (1989). Amplitude, width, and interarrival distributions for noise impulses on intrabuilding power line communication networks. IEEE Trans. Electromagn. Compatibility, 31(3), 320-323.

[9] Benyoucef, D. (2003). A new statistical model of the noise power density spectrum for powerline communications. Proceedings of the 7th International Symposium on Power Line Communications and Its Applications (pp. 136-141)

[10] Bo, W., Yinghao, Q., Peiwei, H., \& Wenhao, C. (2007). Indoor powerline channel simulation and capacity analysis. Proceedings of the IET Conference on Wireless, Mobile and Sensor Networks (pp. 154-157).

[11] Middleton, D. (1977). Statistical-physical models of electromagnetic interference. IEEE Trans. Electromagn. Compat., 19(3), 106-127.

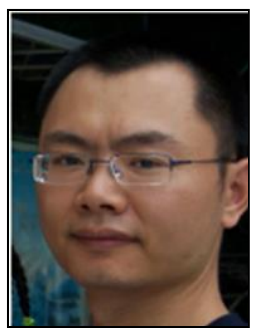

Guoping Chen was born in Chongqing, China, in 1976. He received his PhD degree in electromagnetic field and microwave technology from University of Electronic Science and Technology of China, Chengdu, China, in 2009.

He has studied in the post-PhD study station of College of Communication Engineering at University of Electronic Science and Technology of China from 2009 to 2011. And he was granted a visiting scholar position in Duke University from 2011 to 2012.

He is now a professor at Chongqing University of Posts and Telecommunication. His current research focuses on millimeter-wave electromagnetic imaging, indoor positioning and power line communication.

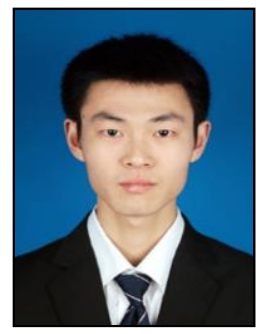

Gonghai Yang was born in Chongqing, China, in 1992. He received his BS degree in electronic science and technology from Chongqing University of Posts and Telecommunications, Chongqing, China, in 2016.

$\mathrm{He}$ is pursuing his master degree with electrical engineering theory and new technology at Chongqing University of Posts and Telecommunications.

His research interests include signal processing and power line communication. 